\title{
The "Journal of Functional Morphology and Kinesiology" Journal Club Series: Highlights on Recent Papers in Movement Analysis
}

\author{
Lingyan Wang ${ }^{1}$, Luís Silva ${ }^{2}$, Michelino Di Rosa ${ }^{3}$, Carlos Marques ${ }^{2}$, João Barreiros ${ }^{2}$ and \\ Jan Cabri $4,5,6$,* \\ 1 Oregon Hearing Research Center, Oregon Health \& Science University, \\ 3181 SW Sam Jackson Park Road/NRC04, Portland, OR 97239, USA; wangli@ohsu.edu \\ 2 Laboratory of Motor Behavior, Faculty of Human Kinetics, University of Lisbon, 1300-344 Lisbon, Portugal; \\ lsilva0a@gmail.com (L.S.); carlos.marques@web.de (C.M.); jbarreiros@reitoria.ulisboa.pt (J.B.) \\ 3 Department of Biomedical and Biotechnology Sciences, University of Catania, Via Androne 83, \\ 95123 Catania, Italy; mdirosa@unict.it \\ 4 Department of Physical Performance, Norwegian School of Sport Sciences, 0863 Oslo, Norway \\ 5 Lunex International University of Health, Exercise \& Sports, L-4671 Differdange, Luxembourg \\ 6 Department of Gerontology, Faculty of Medicine and Pharmacy, Vrije Universiteit Brussel, \\ B-1090 Brussels, Belgium \\ * Correspondence: jan.cabri@nih.no
}

Received: 6 February 2017; Accepted: 6 February 2017; Published: 8 February 2017

\begin{abstract}
We are pleased to introduce the fourth Journal Club. This edition is focused on several relevant studies published in the last years in the field of movement analysis, chosen by our Editorial Board members. We hope to stimulate your curiosity in this field and to share with you the passion for the sport seen also from the scientific point of view.
\end{abstract}

Keywords: tendon injures; posture; movement; muscle exercise; aging; parkinson disease; total knee arthroplasty; return to driving

\section{Introduction}

Human movement analysis is the observation and definition of movements of humans. Movement analysis is often carried out in a laboratory. Simple analysis can involve simple observations. Advanced analysis often involves some form of technology, for example high speed, or optical/optoelectronic cameras to generate the kinematics needed for analysis. Often, force plates and/or electromyography can be combined to provide complete information [1]. The study of movement analyses is an integrated perspective influenced by the fields of motor learning, anatomy, functional morphology, experimental psychology, neuropsychology, kinesiology, biomechanics, and human factors engineering. The level of management is directed toward upperclass undergraduate and graduate students in physical education and others pursuing an analytical understanding of human movement. Human movement includes how the human organism learns to move, the underlying factors leading to the structure of movement, how our movements adapt to simple and complex environmental situations, and how we might proceed in our quest for understanding communication through a multidimensional technique in the analysis of human movement [2]. A structural analysis of human movement is explored as one means of determining the characteristics of movement under differing environmental, morphologic, and biomechanical conditions. The principles of organization of planned, purposeful human movement are developed through an understanding of patterns of human movement, the factors influencing skilled motoric adaption to our environment, and the neuromuscular control processes involved [3]. The movement analysis allows us to verify the ability to perform various movements and to improve 
their efficiency by improving the body schema and coordinative and proprioceptive components of the subject. Analyzing a movement is equivalent to locating the position and speed at each instant and characterizing the linear or angular displacement of each part of the body in motion [4].

There are different types of movement analysis, i.e., the kinematic, dynamic, quantitative and qualitative movement analysis [5]:

- The kinematic analysis studies the movement caused by forces and motion factors applied together. The most important results are the union of motion and definition of displacements, velocities and accelerations of the parties.

- The dynamic motion analysis allows to evaluate the forces generated by the movement, and the movement itself.

- The qualitative analysis describes and analyses movements non-numerically, by seeing movements as "patterns", while quantitative analysis describes and analyses movement numerically.

- The quantitative analysis can sometimes appear more objective because of its "data"; however the accuracy and reliability of such data can be very suspect, particularly when obtained in competition. Qualitative analysis is often more strongly rooted in a structured and multidisciplinary approach, whereas quantitative analysis can appear to lack a theoretical grounding and to be data-driven.

\section{Recent Papers Regarding the Movement Analysis}

\subsection{Gene Therapy Strategies Could Improve Tendon Healing and Benefit Movement}

Highlight by Lingyan Wang

As an important part of the musculoskeletal system, tendons are fibrous connective tissues that connect muscle to bone. Tendon injures frequently happen in daily life. However, clinical therapeutic options are limited to conservative and surgical treatments with a long recovery period. $\mathrm{Wu}$, et al. [6] developed a novel method to augment tendon healing and reduce tendon adhesion through gene therapy. As we know, transforming growth factor beta 1 (TGF- $\beta 1$ ) plays a critical role in adhesion formation during tendon healing. In this study, Adeno-associated virus (AAV) was used to transfer TGF- $\beta 1-$ miRNA to injured and surgically repaired tendons in chickens. Several weeks after AAV-mediated treatment, the improvement of tendon gliding and decreases in adhesion formations were found. These results supported the intraoperative treatment of TGF- $\beta 1$-miRNA for the recovery of tendon function after surgery. AAV is a promising method for gene therapy and widely used in therapeutic applications. Moreover, this study demonstrates that AAV-mediated TGF $\beta 1$-miRNA may be a new strategy for treating tendon injury. Tendon abnormalities have also been described in some inherited metabolism disorders, such as Alkaptonuria which leads to a reduction in the structural integrity of collagen and increases rupture. Recently, Depreux et al. used a novel in utero gene transfer method $[7,8]$ for delivery of active antisense oligonucleotides (ASOs) into amniotic cavity of mouse embryo [9]. The amniotic cavity surrounding the fetus could serve as an ideal drug reservoir. ASOs are an established tool for the therapeutic modulation of gene expression. Fetal therapeutic strategies to manage disease processes represent a powerful new approach for clinical care and open the door for fetal drug therapy to treat congenital disease of the tendon.

\subsection{Age Related-Variations in the Spatial Magnitude of Postural Sway}

\section{Highlight by Luís Silva}

The structure behind the biological organization and its complex interactions, feedback capacity and regulation is characterized by a chaotic structure [10], which is influenced by unhealthy pathological states or skill deficits [11]. When deterministic traditional linear measures were unable to quantify uncertainties in data, nonlinear ones are capable of detecting subtle differences within the 
time series, such as for postural sway. Force-plate posturography has been a common method used to assess postural control [12] measuring anteroposterior (AP) and mediolateral (ML) displacement of the center of pressure (CoP). A recent study [8] reported that the spatial magnitude of sway is affected by the gaze target distance in older adults, also showing that the width of the multifractal spectrum (a measure of temporal dynamics) is greater for older adults when compared with younger adults. Accordingly, Mufano, Wade, Stergiou and Stoffregenet [13] evaluated the kinematics of the $\mathrm{CoP}$, regarding the amount of sway and the multifractality, in elderly people standing on a ship at sea (that departed from Nassau, Bahamas) when looking to the nautical horizon. Two premises were asked: (1) the spatial amplitude of postural sway could decrease when looking at the nautical horizon comparing to a closer target; (2) the nautical horizon could affect the multifractality of standing body sway in older adults. For this purpose, 18 adults aged from 56 to 78 years were recruited and their postural activity was evaluated during stance on a force plate at a frequency of $50 \mathrm{~Hz}$. The authors found that both positional variability and width of the multifractal spectrum were higher in the body's ML axis than in the AP axis. The spectrum width was also higher when looking at the horizon than looking to a nearby target, contrarily to the first premise. Additionally, the target distance variation affected spectrum width for postural activity in the AP axis, but not in the ML axis. These findings showed that the nautical horizon has an influence on the multifractality of sway, increasing spectrum width when regarding a closer target. These results are different from those observed on land where a decreased spectrum width is observed for a more distant visual targets compared to closer ones [14]. The ship motion is seen as a form of motor constraint that can eliminate age related-variations in the spatial magnitude of postural sway. Future research is needed to understand changes in complexity with aging and its adaptive sway capabilities when submitted to different motor constraints.

\subsection{Muscle Exercise and Parkinson Disease (PD)}

\section{Highlight by Michelino Di Rosa}

Parkinson's disease (PD) is a progressive movement disorder diagnosed in $1 \%$ of the US population over age $65[15,16]$. This disease presents as a progressive loss of neurons in the substantia nigra of the midbrain; thus altering the nigrostriatal neural conduction [17]. In PD, the cause of fatigue and decreased endurance is unknown but thought to be associated with disease processes involving injuries to the basal ganglia and Restless Leg Syndrome (RLS). Bradykinesia also increases fatigue by prolonging the time required to complete activities and tasks. The individual must work harder to carry out simple movements or tasks. Muscles do not move well or are poorly conditioned, i.e., atrophy [18]. Loss of muscle strength increases fatigue and decreases endurance [19]. Zhao M. et al. in the manuscript entitled "Effects of coordination and manipulation therapy for patients with Parkinson disease" [20], analyzed the effects of a new exercise training regimen, i.e., coordination and manipulation therapy (CMT), on motor, balance, and cardiac functions in patients with Parkinson disease (PD). They divided 36 PD patients into the CMT $(n=22)$ and control $(n=14)$ groups. The patients in the CMT group performed dry-land swimming (imitation of the breaststroke) and paraspinal muscle stretching for $30 \mathrm{~min} /$ workday for 1 year. The control subjects did not exercise regularly. The same medication regimen was maintained in both groups during the study. Clinical characteristics, Unified Parkinson's Disease Rating Scale (UPDRS) scores, Berg balance scale (BBS) scores, mechanical balance measurements, timed up and go (TUG) test, and left ventricular ejection fraction (LVEF) were compared at 0 (baseline), 6 , and 12 months. Biochemical test results were compared at 0 and 12 months. The primary outcome was motor ability. The secondary outcome was cardiac function. In the CMT group, UPDRS scores significantly improved, TUG test time and step number significantly decreased, BBS scores significantly increased, and most mechanical balance measurements significantly improved after 1 year of regular exercise therapy (all $p<0.05$ ). In the control group, UPDRS scores significantly deteriorated, TUG test time and step number significantly increased, BBS scores significantly decreased, and most mechanical balance measurements significantly worsened after 1 year (all $p<0.05)$. LVEF 
improved in the CMT group only $(p=0.01)$. This preliminary study suggests that CMT effectively improved mobility disorder, balance, and cardiac function in PD patients over a 1-year period.

\subsection{Return to Safe Driving after Total Knee Arthroplasty: More Kinesiological Research Is Needed}

Highlight by Jan Cabri, Carlos Marques and João Barreiros

Total knee arthroplasty (TKA) is a common orthopaedic surgical procedure with high success and long-term survival rates. The features of enhanced rehabilitation programs (sometimes also called fast-track protocols) are in-depth patient education; modified anaesthesia protocols; early mobilization (same-day mobilization); rapid resumption of activities of daily living; and multimodal pain therapy. A positive effect of this approach is a reduction in length of hospital stay (LOS), without an increase in readmission rates due to complications (LOS reductions from 10 to 4 days have been reported). Despite the existence of well-structured information about the ongoing rehabilitation process, patients want to know when they can safely resume car driving. The question is of socio-economic importance, since some patients are discharged from the hospital to their homes and depend on driving a car to reach the doctor or the rehabilitation center. However, the available evidence on which physicians and doctors can rely when advising patients on when they can safely resume car driving after TKA is scarce.

An important human factor in accident prevention research is the brake response time (BRT). The BRT is a measure of cognitive and psychomotor performance and has been used in traffic accident prevention research to assess driving capability in different populations. BRT can be divided into two main components: reaction time (RT) and movement time (MT). RT is defined as the time frame needed for signal perception, signal identification and response selection. Accordingly, RT ends with the initiation of the motor component of the response, that is, with the early evidence of muscle activity or with a pressure reduction on the gas pedal. In contrast, MT is defined as the motor response to the signal.

TKA causes MT delay, which affects BRT negatively. An increase in task complexity also significantly increases BRT [21-23]. TKA seems to affect peripheral aspects related to the execution of the movement. Soft tissue lesions may be the cause of such performance impairments after TKA.

However, a review of the literature revealed that the normalization of BRT after TKA varied among the 10 existing studies and ranged from 28 to 56 days. Multiple factors may have led to the wide variation of results. Methodological weaknesses in some studies and the long time period between the first and the last publication with all the developments made in the field (i.e., surgery techniques, anaesthesia protocols, rehabilitation protocols) are two possible explanations. In view of the discrepancy of results it is not possible to make a generalized evidence-based recommendation. Further high quality research on this issue is necessary.

Conflicts of Interest: The authors declare no conflict of interest.

\section{References}

1. Wong, W.Y.; Wong, M.S.; Lo, K.H. Clinical applications of sensors for human posture and movement analysis: A review. Prosthet. Orthot. Int. 2007, 31, 62-75. [CrossRef] [PubMed]

2. Musumeci, G. Welcome to the New Open Access Journal of Functional Morphology and Kinesiology. J. Funct. Morphol. Kinesiol. 2016, 1, 1-5. [CrossRef]

3. Lu, T.W.; Chang, C.F. Biomechanics of human movement and its clinical applications. Kaohsiung J. Med. Sci. 2012, 28, S13-S25. [CrossRef] [PubMed]

4. Cappozzo, A.; Della Croce, U.; Leardini, A.; Chiari, L. Human movement analysis using stereophotogrammetry. Part 1: Theoretical background. Gait Posture 2005, 21, 186-196. [PubMed]

5. Della Croce, U.; Leardini, A.; Chiari, L.; Cappozzo, A. Human movement analysis using stereophotogrammetry. Part 4: Assessment of anatomical landmark misplacement and its effects on joint kinematics. Gait Posture 2005, 21, 226-237. [CrossRef] [PubMed] 
6. Wu, Y.F.; Mao, W.F.; Zhou, Y.L.; Wang, X.T.; Liu, P.Y.; Tang, J.B. Adeno-associated virus-2-mediated TGF- $\beta 1$ microRNA transfection inhibits adhesion formation after digital flexor tendon injury. Gene Ther. 2016, 23, 167-175. [CrossRef] [PubMed]

7. Wang, L.; Jiang, H.; Brigande, J.V. Gene transfer to the developing mouse inner ear by in vivo electroporation. J. Vis. Exp. 2012, 64, e3653. [CrossRef] [PubMed]

8. Jiang, H.; Wang, L.; Beier, K.T.; Cepko, C.L.; Fekete, D.M.; Brigande, J.V. Lineage analysis of the late otocyst stage mouse inner ear by transuterine microinjection of a retroviral vector encoding alkaline phosphatase and an oligonucleotide library. PLOS ONE 2013, 8, e69314.

9. Depreux, F.F.; Wang, L.; Jiang, H.; Jodelka, F.M.; Rosencrans, R.F.; Rigo, F.; Lentz, J.J.; Brigande, J.V.; Hastings, M.L. Antisense oligonucleotides delivered to the amniotic cavity in utero modulate gene expression in the postnatal mouse. Nucleic Acids Res. 2016, 44, 9519-9529. [PubMed]

10. Stergiou, N.; Harbourne, R.T.; Cavanaugh, J.T. Optimal Movement Variability. J. Neurol. Phys. Ther. 2006, 30, 120-129. [CrossRef] [PubMed]

11. Stergiou, N.; Decker, L.M. Human movement variability, nonlinear dynamics, and pathology: Is there a connection? Hum. Mov. Sci. 2011, 30, 869-888. [CrossRef] [PubMed]

12. Błaszczyk, J.W. The use of force-plate posturography in the assessment of postural instability. Gait Posture 2016, 44, 1-6. [CrossRef] [PubMed]

13. Munafo, J.; Curry, C.; Wade, M.G.; Stoffregen, T.A. The distance of visual targets affects the spatial magnitude and multifractal scaling of standing body sway in younger and older adults. Exp. Brain Res. 2016, 234, 2721-2730. [CrossRef] [PubMed]

14. Munafo, J.; Wade, M.G.; Stergiou, N.; Stoffregen, T.A. The Rim and the Ancient Mariner: The Nautical Horizon Affects Postural Sway in Older Adults. PLoS ONE 2016, 11, e0166. [CrossRef] [PubMed]

15. Gelb, D.J.; Oliver, E.; Gilman, S. Diagnostic criteria for parkinson disease. Arch. Neurol. 1999, 56, $33-39$. [CrossRef] [PubMed]

16. Borg, G. Physical training. 3. Perceived exertion in physical work. Lakartidningen 1970, 67, 4548-4557. [PubMed]

17. Agid, Y. Parkinson's disease: Pathophysiology. Lancet 1991, 337, 1321-1324. [CrossRef]

18. Milanovic, S.; Filipovic, S.R.; Radovanovic, S.; Blesic, S.; Ilic, N.V.; Kostic, V.S.; Ljubisavljevic, M.R. Changes in motor cortex excitability associated with muscle fatigue in patients with Parkinson's disease. Vojnosanitetski pregled 2013, 70, 298-303. [CrossRef] [PubMed]

19. Nirenberg, M.J. Dopamine agonist withdrawal syndrome: Implications for patient care. Drugs Aging 2013, 30, 587-592. [CrossRef] [PubMed]

20. Zhao, M.; Hu, C.; Wu, Z.; Chen, Y.; Li, Z.; Zhang, M. Effects of coordination and manipulation therapy for patients with Parkinson disease. Int. J. Neurosci. 2016. [CrossRef] [PubMed]

21. Marques, C.; Cabri, J.; Barreiros, J.; Carita, A.; Friesecke, C.; Loehr, F. The effects of task complexity on brake response time before and after primary total knee arthroplasty. Arch. Phys. Med. Rehabil. 2008, 89, 851-855. [CrossRef] [PubMed]

22. Marques, C.; Cabri, J.; Barreiros, J.; Carita, A.; Friesecke, C.; Loehr, F. Does the brake response time of the right leg change after left total knee arthroplasty? A prospective study. Knee 2008, 15, 295-298. [CrossRef] [PubMed]

23. Marques, C.; Santos, R.; Gamboa, H.; Lampe, F.; Barreiros, J.; Cabri, J. Brake response time before and after total knee arthroplasty-Tracking possible effects of the surgery technique on motor performance: Report of two cases. In Proceedings of the International Conference on Bio-inspired Systems and Signal Processing, Algarve, Portugal, 1-4 February 2012; pp. 419-422.

(C) 2017 by the authors; licensee MDPI, Basel, Switzerland. This article is an open access article distributed under the terms and conditions of the Creative Commons Attribution (CC BY) license (http:/ / creativecommons.org/licenses/by/4.0/). 